\title{
A Critical Review of Optimization Methods for Road Vehicles Design
}

\author{
Massimiliano GOBBI ${ }^{1}$ \\ Department of Mechanical Engineering. Politecnico di Milano (Technical University), Milan, ITALY \\ Imtiaz HAQUE ${ }^{2}$ \\ Department of Mechanical Engineering. Clemson University, Clemson, USA. \\ Panos P.Y. PAPALAMBROS ${ }^{3}$ \\ Optimal Design Laboratory. The University of Michigan, Ann Arbor, USA \\ Giampiero MASTINU ${ }^{4}$ \\ Department of Mechanical Engineering. Politecnico di Milano (Technical University), Milan, ITALY.
}

\begin{abstract}
[Abstract] The paper deals with the optimal design of road vehicles and their subsystems. The highly competitive nature of the automotive industry demands constant product innovation and reduction in product development both in time and cost while satisfying performance and legal requirements. Optimization procedures provide a scientific approach to automatically determine the most efficient designs under the target operating environment. Particular attention is devoted to the class of methods pertaining to complex system design optimization, as well as the approaches for the optimal design of complex systems under uncertainty. A critical review of the optimization methods used for solving road vehicle system design problems is reported. Some examples of design optimization are given in the field of vehicle system dynamics, powertrain design, internal combustion engine design, active and passive safety, vehicle system design and lightweight structures, advanced automotive electronics.
\end{abstract}

\section{Introduction}

The design process is and will always be based on the designer intuition. However, optimization techniques can permit significant improvement in the process itself. The designer activity undergoes iterations between an analysis stage, in which the relationships between parameters, performance indices and design constraints are assessed, and a synthesis stage, in which the possible and desired performances are obtained by a proper parameter setting. The inherently iterative nature of the design process makes it particularly difficult to keep it under control.

The application of optimization in automotive engineering is increasing as more powerful computers become available. Today calculations can be performed in a fraction of the time it took a few years ago. Therefore, the applications for numerical optimization have increased.

\footnotetext{
${ }^{1}$ Department of Mechanical Engineering. Politecnico di Milano (Technical University), Via La Masa, 34, I-20156 Milan, ITALY, AIAA Member (Corresponding Author's e-mail: massimiliano.gobbi@polimi.it).

${ }^{2}$ Department of Mechanical Engineering. Clemson University, 106-B Fluor Daniel Building, Clemson, SC 29634-0921, USA.

${ }^{3}$ Optimal Design Laboratory. University of Michigan, 2250 G.G. Brown Building, Ann Arbor, MI 48109, USA.

${ }^{4}$ Department of Mechanical Engineering. Politecnico di Milano (Technical University), Via La Masa, 34, I-20156 Milan, ITALY.
} 
It is assumed in the following, that an accurate (validated) mathematical model of the system under optimization is available. Successful optimization requires availability of appropriate analysis models and knowledge of the capabilities and limitations of the mathematical optimization techniques. ${ }^{6,76,83,67}$

In real-world applications, problems with a single, well-defined objective function tend to be the exception rather than the rule. In engineering, most optimization problems are multi-objective in their nature. In a problem with multiple objectives, it is generally impossible to obtain a ranking of all of the alternative solutions, without using further rules or assumptions. In this situation, the decision of the better solution may become subjective, or must rely on additional information, such as the importance of each objective. The most common and rigorous approach to overcome this limitation is to define the efficient solutions set. ${ }^{9,39,69}$ If possible, the designer has to identify hierarchical levels in the complex system under study so the system can be divided into subsystems that can be further subdivided. Analytical target cascading is a methodology for optimal design of hierarchical multilevel systems where outputs of lower-level elements are the inputs to higher-level elements. ${ }^{54,57,75}$ In any case, optimization of complex systems may require a prohibitive simulation effort, even with the most efficient techniques. In this case, the relationship between design variables and objective functions is expressed by means of a global approximation approach. The physical model is substituted by another purely mathematical model, which is able to give computational results very quickly. ${ }^{29,44,70}$ The inclusion of uncertainty in the problem formulations is a

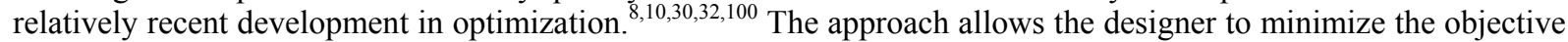
function while accounting for the influence of stochastic variations of some parameters and/or variables. Typically this involves trading (deterministic) optimality for robustness, whether in the value of the objective (robust design) or in the satisfaction of the constraints (reliable design). After the optimal solutions have been computed, ${ }^{11,44,93,69}$ the designer can make a choice and select from this set the preferred solution featuring the desired compromise among objective functions. ${ }^{18}$

A brief outline of optimal design methods generally used for vehicle design problems is given below. Some examples of design optimizations will be given in the field of vehicle system dynamics, powertrain/internal combustion engine design, active safety and ride comfort, vehicle system design and lightweight structures, and advanced automotive electronics. ${ }^{9,23,27,39,54,71,88}$

\section{BASICS}

In this section some basic concepts of optimization theory are reported. The interested reader may refer to Refs. 83, $76,6,84,67$ for an in-depth documentation on these topics.

\section{A. Formulation of the optimization problem}

An optimal design problem is defined by the design variables vector $x$, and a set of performance attributes (objective functions) and design constraints. The majority of engineering problems involve a constrained optimization formulation, that is, the task of minimizing (or maximizing) a vector of objective functions subject to different types of constraints.

Objective functions and constraints must be quantifiably expressed as functions $(f, g)$ of the design variables $x$, i.e., find $x$ such that

$\min _{x \in R^{n}} f(x)$

$h_{j}(x)=0, \quad j=1 \ldots, m_{e q}$

$g_{j}(x) \leq 0, \quad j=1 \ldots, m_{\text {ineq }}$

$x \in X$

where $f$ is the objective function vector, $x$ is the vector of the design variables, $X$ is the definition domain of $x$, and $h_{j}$, $g_{j}$ are equality and inequality constraints, respectively. The set $X$ can represent certain ranges of real values or certain types, such as integer or "standard" values, which are very often used in design specifications. ${ }^{83,76}$

Multi-objective optimization methods can be broadly decomposed into two categories: Scalarization approaches and Pareto approaches. Several methods exist for converting the multi-objective formulation into one with a scalar objective function that can be solved with the usual single objective optimization methods, typically using some sort 
of weighting function. The shortcoming of such scalarization is that the designer must include subjective information and preferences a priori, i.e., before the results of the optimization process are known. Additionally, some optimal solutions can be unattainable for non convex problems (see Refs. 83, 76, 67).

The Pareto methods, on the other hand, keep the elements of the objective vector $f$ separate throughout the optimization process. The most common and rigorous approach is to define the efficient solutions (i.e. Pareto Optimal Solutions). Under this assumption the set of efficient solutions constitutes the Pareto-optimal set. ${ }^{67,69}$ The $^{-1}$ main disadvantage of this approach is that generating Pareto-optimal sets is generally computationally expensive.

The Pareto-optimal solutions are defined mathematically as follows. Given the minimization problem (1) with $k$ objective functions and $n$ design variables, a solution $x_{i}$ is Pareto-optimal if there is no solution $x_{j}$ such that

$$
\left\{\begin{array}{c}
f_{m}\left(x_{j}\right) \leq f_{m}\left(x_{i}\right) \quad m=1,2,3, \ldots, k \\
\exists l: f_{l}\left(x_{j}\right)<f_{l}\left(x_{i}\right)
\end{array}\right.
$$

This definition can be used to find the Pareto-optimal solutions directly (Pareto filtering, see Table 1). ${ }^{69}$

Table 1 provides a list of Multi-objective Optimization methods. An overview of these methods can be found in Refs. 17, 67. Some scalarization methods can also be used to generate the Pareto-optimal set.

\begin{tabular}{|l|l|}
\hline Scalarization methods & Pareto methods \\
\hline Weighted sum (linear and non-linear) & Pareto filtering \\
\hline Constraints method & Adaptive weighted sum method \\
\hline Multiattribute Utility Analysis & Normal Boundary Intersection \\
\hline Goal programming & Multi-objective Evolutionary Algorithms \\
\hline$\ldots \ldots$ & $\ldots \ldots$ \\
\hline
\end{tabular}

Table 1. Overview of multi-objective optimization methods.

\section{B. The solution methods}

The problem in Eq. (1) is formally called a mathematical programming problem. ${ }^{6,76,83,84}$ If the functions $f$ and $g$ are all linear, the problem is a linear programming one. Otherwise, the problem is a non-linear programming one. A large class of design problems comprises mixed-discrete/continuous design variables values. ${ }^{84}$

In general, the solution methods can be divided into derivative and non-derivative methods, see Table 2. Nonderivative methods, are more suitable for general engineering design problems, but the computational efficiency can be limited.

In Table 2, some of the methods for solving optimization problems are presented and compared. ${ }^{67,64}$ This topic is further discussed in the following sub-sections.

\begin{tabular}{|c|c|c|c|c|c|}
\hline Method & Type & $\begin{array}{c}\text { Computational } \\
\text { efficiency }\end{array}$ & \multicolumn{2}{|c|}{$\begin{array}{c}\text { Accuracy } \\
\text { Discrete design } \\
\text { variables values } \\
\text { allowed }\end{array}$} \\
\hline Exhaustive & non-derivative & -- & $\begin{array}{c}++ \\
\text { (discrete } \\
\text { variables) }\end{array}$ & $\begin{array}{c}- \\
\text { (continuous } \\
\text { variables) }\end{array}$ & Yes \\
\hline Uniformly distributed sequences & non-derivative & - & \multicolumn{2}{|c|}{-} & Yes \\
\hline Evolutionary strategies & non-derivative & + & \multicolumn{2}{|c|}{+} & yes \\
\hline $\begin{array}{c}\text { Sequential Quadratic } \\
\text { Programming }\end{array}$ & derivative & ++ & & no \\
\hline
\end{tabular}

Table 2. Methods for solving optimization problems (- - very bad; - poor; + good; + + very good ). 


\section{Nonlinear programming}

The reader may refer to Refs. 83, 76, 6, 84 for a detailed description of nonlinear programming methods.

\section{Search based on uniformly distributed sequences}

The exhaustive search method is the simplest search method, but it requires a huge amount of computational effort. ${ }^{69}$ Design space exploration is a collection of statistical techniques providing a systematic way to sample the design space. In multi-dimensional spaces, a low discrepancy sequence can be used to define a set of design variables combinations to be exploited to sample the objective functions domain by reducing as much as possible the number of combinations. ${ }^{97}$ Low discrepancy sequences differ from pseudo-random sequences in that the points are more evenly distributed in the feasible space.

\section{Evolutionary Strategies}

Evolutionary strategies are a class of non-gradient methods which has grown in popularity since Holland first published his book on the subject. ${ }^{48}$ Two types of evolutionary algorithms are considered here, namely genetic algorithms and simulated annealing.

Genetic algorithms (GAs) are suitable for finding the minimum of a function (or of a set of functions) by performing a semi-stochastic search. ${ }^{11,15,41,48,87,93}$ The basic idea of GAs is the mechanics of natural selection. The design variable vector referring to a particular design solution is typically encoded into binary or real strings (chromosomes). GAs are based on an elitist reproduction strategy, where the strongest members of the population (design solutions) are selected for reproduction and are given the opportunity to strengthen the chromosomal (i.e., genes, namely, design variables) makeup of the next generation. Unlike many other search techniques, GAs consider multiple design solutions (a population) at each iteration. The main advantages of GAs are the following. GAs work on function evaluations alone and do not require function derivatives. GAs proceed from several points in the design variable domain (population), consequently, the method has a better chance of locating the global minimum. GAs allow design variable spaces consisting of a mix of continuous and discrete variables. GAs can be easily implemented on parallel computers. The disadvantage is that the computational costs are high, and good performance often requires a difficult tuning of the algorithm for the specific problem at hand. A ranking method can be easily included to grade the population in terms of Pareto-optimality and construct a procedure able to identify the entire Pareto optimal set and not, as it occurs for standard algorithms, one single Pareto-optimal solution for each run. ${ }^{11,16,41,93}$

Simulated annealing (SA) was first presented by Kirkpatrick (see Ref. 40) in the early 80's. Simulated annealing is a heuristic search method suitable to solve difficult optimization problem. SA simulates the natural phenomenon of annealing of solids in order to optimize complex systems. Annealing of solids is accomplished by heating up a solid and allowing it to cool down slowly so that thermal equilibrium is maintained. This ensures that the atoms are obtaining a minimum energy state. ${ }^{52,84}$ Annealing is the process that refers to finding a low energy state of a solid by initially melting the substance and then lowering the temperature slowly. At high temperatures the molecules of a liquid move freely with respect to each other. If the liquid is cooled slowly, the thermal mobility is lost, the atoms are able to line themselves up and form a pure crystal structure that is completely ordered in all direction. This crystal structure corresponds to the state of minimum energy of the system. Simulated annealing can be seen as a subset of Genetic Algorithms with a population of one single individual and a changing mutation rate.

In 1953 Metropolis, ${ }^{68}$ introduced a simple algorithm to simulate the annealing process by considering a collection of atoms at a given temperature. At each iteration an atom is given a small random displacement and the resulting change in energy $(\delta)$ of the system is calculated. If $\delta<0$, the resulting change is accepted. If $\delta>0$, the change is accepted with probability $\exp \left[\delta /\left(K_{b} T\right)\right]$, where $T$ is the temperature and $K_{b}$ is the Boltzmann constant. If a large number of iterations is carried out at each temperature, the system attains thermal equilibrium at each temperature. At thermal equilibrium the probability distribution of the system follows the Boltzmann distribution, where the probability of the system being in a state $i$ at temperature $T$ is $\exp \left(-E_{j}\right) /\left(K_{b} T Z\right)$, where $E_{i}$ is the energy of the state $i$ and $Z$ is the partition function required for normalization. The above statement expresses the idea that a system in thermal equilibrium at temperature $T$ has its energy probabilistically distributed among all different energy state $E$. Even at low temperature there is a chance of the system being in high energy state, therefore there is a corresponding chance for the system to get out of a local minimum energy state in favor of finding a better global one.

To apply Metropolis algorithm for other than thermodynamic process one must provide the following

(a) A description of possible system configurations. 
(b) A generation of the random changes in the configurations.

(c) An objective function $E$, whose minimization is the goal.

(d) A control parameter $T$ and an annealing schedule.

There has been some work done in multi-objective simulated annealing where the non-dominated solutions found during search are stored during the annealing process. ${ }^{95}$

\section{Global approximation}

Complex system design requires extensive use of simulation-based design and analysis tools, such as multibody dynamics simulations (MBD), finite element analysis (FEA), computational fluid dynamics (CFD), and other computationally intensive models. The need to quantify the engineering performance of complex products, such as automobiles, leads to computationally expensive models due to fidelity of modeling and to the obvious need for accurate results.. Moreover, computer simulation codes can be discipline-specific, distributed, on different computer platforms. Sometimes, computationally expensive computer simulations and/or analyses are replaced by surrogate models, which are fast, simple approximations. To approximate functions, a vast series of techniques exist that go from a simple polynomial interpolation to very more accurate methods of representing function behavior, such as radial base neural networks or kriging models. ${ }^{85}$

Optimization may require a prohibitive simulation effort, even with the most efficient techniques. In this case, the original model can be globally approximated by means of a simpler, approximate model. This approximate model is defined on the basis of a limited number of simulations (i.e., computational experiments) performed by means of the originally validated physical model of the system under consideration. Typically the simulation time for the pure numerical model is a small fraction $(1 / 10$ to $1 / 10000)$ of the simulation time needed by the original model. ${ }^{73,70}$ The accuracy in the approximation of the outputs depends on the approximation model employed and the computational effort expended to build the model. The first attempts using neural networks for global approximation were reported in Ref. 44. A comparison of different approximation methods is given in Ref. 29 and some results are shown in Table 3.

\begin{tabular}{|c|c|c|c|c|}
\cline { 2 - 4 } \multicolumn{1}{c|}{} & \multirow{2}{*}{$\begin{array}{c}\text { approximation } \\
\text { domain }\end{array}$} & \multicolumn{2}{|c|}{ evaluation accuracy } & \multirow{2}{*}{$\begin{array}{c}\text { tuning } \\
\text { effort }\end{array}$} \\
\cline { 3 - 4 } & local/global & + & - & ++ \\
\hline $\begin{array}{c}\text { Response surface } \\
\text { model }\end{array}$ & $\begin{array}{c}\text { complex } \\
\text { model }\end{array}$ & \\
\hline Neural Networks & Global & ++ & ++ & - \\
\hline Kriging & Global & ++ & + & - \\
\hline
\end{tabular}

Table 3. Approximation methods. ( - poor; + good; ++ very good ).

\section{Optimal design of multilevel systems}

Analytical target cascading (ATC) is a methodology for optimal design of hierarchical multilevel systems where outputs of lower-level elements are inputs to higher-level elements (See Fig. 1). Every system is analyzed at the level of complexity that corresponds to the interest of the designer. For this reason, we can identify hierarchical levels in the system definition; the system can be divided into subsystems that can be further subdivided.

The objective is to identify the element interactions early in the design process, and to determine specifications that yield consistent system design with minimized deviation from design targets. This is because the time and cost to correct problems increase rapidly with each step of the product development cycle. The process formulates and solves a minimum deviation optimization problem for each element of the multilevel system. By solving the element optimization problems in an iterative manner, ATC aims at minimizing discrepancies between the optimal design variable values desired at elements higher in the hierarchy and response values that elements at lower levels can actually deliver. ${ }^{51,83,55,75,74}$ In addition, if design variables are shared among some elements at the same level, their required common final optimal value is coordinated by their parent element at the level above. 


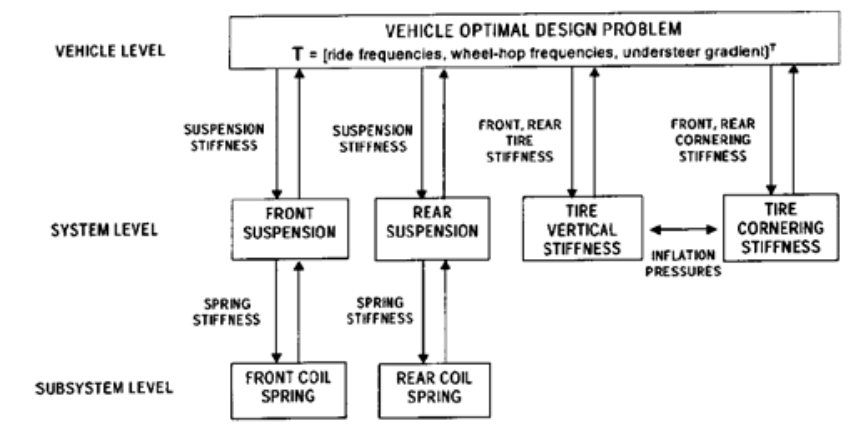

Fig. 1 Example of hierarchical multilevel system decomposition, adapted from Ref. 51.

\section{Comparison of the different solution methods}

There is no unique answer to which optimization method is the best. Many studies have shown that different methods show the best performance depending on the problem and on how much effort has been spent to tune the method itself (the procedure itself needs to be optimized).

Search based on uniformly distributed sequences can be computationally very expensive, but no parameters relative to the algorithm itself need to be tuned. Genetic Algorithms are robust and they can handle multi modal functions, but several parameters (population size, mutation and crossover probability, ...) need to be tuned to get adequate convergence properties. Simulated Annealing is a robust method slightly less computational expensive then genetic algorithms.

Standard nonlinear programming methods show very fast convergence rate and they are very easy to be tuned, but they are not as robust (i.e., get stuck in local optima) as the other methods. Moreover, they can just find one optimal solution for each run. Moreover, the solution of engineering problems often requires a mixture of numerical simulations, analytical calculations and selection of catalog components. Therefore, non-gradient optimization methods (i.e., search based on uniformly distributed sequences, genetic algorithms, simulated annealing) are better suited for these types of problems, being impossible the calculation of the derivatives of the objectives function.

\section{Reliability-based design optimization}

Because deterministic optimum designs obtained without taking uncertainty into account could lead to unreliable designs, a reliability-based approach to design optimization is preferable. Recently many authors have introduced procedures that combine design optimization techniques with reliability-based design methods. ${ }^{3,92,8,37,40,61,56,100,91,101}$ The approach allows the designer to minimize the objective function while accounting for the influence of stochastic variations of some parameters and/or variables. Typically this involves trading (deterministic) optimality for robustness, whether in the value of the objective (robust design) or in the satisfaction of the constraints (reliable design).

In the presence of random variables and parameters, responses, such as objective and constraint value calculations, are themselves random variables, whose expected values and/or variances must be computed when solving probabilistic optimization problems. Evaluation of probability distributions of responses can be achieved using Monte Carlo simulation, but this is a very expensive process. Thus, various approximation schemes must be used in practical situations.

A mean-value first-order second-moment approach (first-order Taylor expansion about the current design) can be adopted to estimate the mean and standard deviation of the objective functions. ${ }^{40}$ When a trained artificial neural network is used to compute model responses, an accurate estimate of the variance of the objective functions can be obtained with no additional computational cost. ${ }^{30,40}$

Uncertainty can also come from considering market behavior when launching new products. For example, developing a new vehicle design in a dual-use context (commercial enterprise and government military uses) is affected by uncertainty in market penetration and cannibalization. ${ }^{13}$ 


\section{OPTIMAL DESIGN OF ROAD VEHICLES AND SUBSYSTEMS}

The following state of the art review is adapted from Ref. 28 and is reported here in a revised and extended form.

\section{A. Vehicle system dynamic behavior}

The first attempts to design optimally the suspension system of a road vehicle appeared in Refs. 14, 47, 49, 50, 63, 99, 77, 90. Some basic studies on the optimization of the ride comfort and active safety of road vehicles are presented in Refs. 35, 36, 33, 78. Both passively and actively suspended vehicles have been considered. Simple analytical formulae for the estimation of vehicle dynamic behavior are derived, and these formulae are used in conjunction with multi-objective programming (MOP) to find the optimal suspension design variables symbolically, ensuring the best compromise among comfort, road holding and working space. In Refs. 32, 33 the design variables, i.e., suspension stiffness and damping, are considered as stochastic variables and the vehicle body mass and tire radial stiffness as stochastic parameters, in order to derive the optimal trade-off solutions in a optimal and robust framework.

Referring to the tuning of gains of an actively suspended vehicle, analytical formulae seem to have been derived only for simple system models (results reported in Refs., 2, 49, 98, 36), establishing the well-known 'sky-hook' control strategy. A basic study on the optimization of the handling behavior and active safety of road vehicles is presented in Ref. 71. That article demonstrates how the front and rear tire cornering stiffness of a vehicle can be tuned in order to obtain a preferred compromise among the many conflicting performance indices that describe the handling behavior and active safety. Similar studies in optimizing vehicle handling behavior over a range of maneuvers but involving a much greater number of design variables is reported in Refs. 31, 39, 65, 72, 86, 88, 89, 96. In order to design a car effectively, one is often required to tune the entire set of design variables related to tires, aerodynamics and chassis characteristic (stiffness, damping and kinematics of the suspension system). Such tuning presents good opportunities for effective use of optimization methods. ${ }^{40}$

\section{B. Powertrain design}

Hybrid-electric propulsion systems have been optimized with respect to fuel economy, ride, and mobility targets in Ref. 54 by applying a target cascading approach.

A method for combined design optimization and power management of a hybrid propulsion system integrated within a complete vehicle system was proposed in Ref. 24. A sequential quadratic programming (SQP) algorithm with a design space exploration based multi-start technique was used to enhance the chances of achieving the global optimum.

The design of the synchronizer and selector mechanism of a vehicle gearbox is discussed in Refs. 37, 38. The main aim there is to improve shifting ability during a reference shift action. Fifty-eight variables of the system model were thus tuned. The approach proposed in Ref. 37 addresses both the optimization of nine system performance indices and the reduction of the sensitivity (variance) of the performance indices to stochastic perturbations. The variances are computed by means of a procedure based on the global approximation of the objective functions.

The use of ATC in vehicle transmission design was reported in Ref. 7. ATC was applied to the simulation-based design of a CVT implemented in a mid-sized truck. The overall system was decomposed into three levels. The study highlighted two main issues. First, the development of the co-ordination strategy and appropriate models appeared to be an intricate task that benefits from extensive knowledge of the overall problem. Second, the values of the arbitrary weights defined at all levels had a significant impact on the results and of ATC as a practical and suitable approach.

\section{Internal Combustion Engine design}

A probabilistic formulation of the ATC process has been used in Refs. 57, 10 to solve an internal combustion engine design problem. An engine is considered at the top level system, which is then decomposed into subsystems representing the piston-ring/cylinder-liner subassembly of the cylinders. The system model predicts engine performance in terms of brake-specific fuel consumption. The ring/liner subassembly simulation takes as inputs the surface roughness of the ring and the liner (assumed to be normally distributed) and the Young's modulus and 
hardness and computes power loss due to friction. The engine simulation takes then as input the power loss and computes brake-specific fuel consumption of the engine.

An analysis of an optimized approach for designing engine mounts is given in Ref. 1. GAs and SQP have been used for selecting the combination of parameters that can yield the deepest notch and shortest resonance peak in the dynamic stiffness of the fluid mounts.

The link between manufacturing process and product performance is studied in order to construct quantifiable criteria for the introduction of new engine technologies and processes in Ref. 60. Cost associated with a new process must be balanced against increases in engine performance. A predictive engine simulation model is used to quantify performance gains due to the new surface finish obtained by means of abrasive flow machining for finishing the inner surfaces of intake manifolds for two engines. Subsequently, economic cost-benefit analysis is used to evaluate manufacturing decisions based on their impact on the firm's profitability.

\section{Safety and ride comfort}

In Ref. 5, GAa are used for determining the active control and passive mechanical parameters of a vehicle suspension system in order to minimize the vertical acceleration subject to constraints on road holding and suspension working space. In Ref. 27 a MOP approach using GAs is employed for the design of a multi-link suspension, based on performance indices related to vehicle handling and stability.

In Ref. 72 a procedure is presented for the integrated design (tuning) of tires and suspensions of a racing car. Proper objective functions are defined after a subjective-objective correlation analysis. Different driving situations (steady state, J-turn, lane-change, power on-off while steering, braking on a bend, passing over a kerb while steering) are optimized with respect to 18 design variables related to the suspension system and the tire characteristics. A global approximation model was used together with a search method based on low discrepancy sequences. The same approach has been followed in Refs. 86, 89, 14 and in Ref. 31 for the problem of the chassis design of a production car. In Ref. 39 the computation of the Pareto-optimal set is performed, for a similar chassis design problem by using GAs. In Ref. 40 a method for the reliability-based multi-objective design optimization of tires and suspensions of a racing car is introduced and applied. The optimization has been performed on the basis of a global approximation procedure.

In Ref. 42, through the use of an optimization algorithm with finite-difference sensitivities, the suspension characteristics have been optimized. The ride optimization is achieved by finding the optimum of a ride index that results from a metric that accounts for the acceleration in several key points in the vehicle properly weighted. The extension by including of the optimization of the handling characteristic is given in Ref. 43 .

The design of heavy vehicles for good dynamic performance in a variety of scenarios or maneuvers (from standard test maneuvers to extreme emergency maneuvers) is discussed in Ref. 9. The problem is formulated as a multicriteria, multi-scenario design problem with the goal of finding an optimal vehicle design, which can improve the vehicle dynamic performance in all the considered scenarios simultaneously (lane change, pulse steer, and ramp steer, induced roll-over). A modified Monte-Carlo optimization technique is used to find the optimal designs.

A robust design approach for improving vehicle crashworthiness was applied using the Monte Carlo simulation method on a global response surface in Ref. 45.

In Ref. 91 a methodology for reliability-based multi-objective design optimization of automotive body components under impact scenarios is introduced. The approach is applied to minimize the structural mass while maximizing energy absorption of an automotive rail section under structural and occupant safety constraints.

In Ref. 53 the focus is on design optimization for safety and NVH. The set of vehicle system design variables is tuned in order to minimize the vehicle weight while satisfying constraints on the static torsion and bending displacements and limiting the injury criteria during frontal crash and side impact.

Analytical target cascading has been used to optimize ride quality and handling performances by considering suspension, tire, and spring analysis models in Refs. 51, 55, 56. Potential incompatibilities among targets and constraints throughout the entire system can be uncovered and the trade-offs involved in achieving system targets under different design scenarios can be quantified.

The problem of choosing a single solution within a set of Pareto-optimal alternatives is addressed in Ref. 59. Two methods, the k-optimality approach and the more general k- $\varepsilon$-optimality method, were considered. These two methods theoretically justify and mathematically define the designer's tendency to choose solutions that are "in the middle" of the Pareto-optimal set. The methods were successfully applied to the optimization of the tire/suspension system of a car. 


\section{E. Vehicle system design and lightweight structures}

The optimal layout of structural components is discussed in many papers. ${ }^{58,46,34,62}$ The problem of the definition of the layout of the entire vehicle is addressed in recent papers. ${ }^{46}$ Real-coded genetic algorithms (GAs) have been used to solve the multi-objective optimization problem employing an elitist search.

An efficient way to find good lightweight designs for vehicle structures is to use different tools for structural optimization. ${ }^{94,23,81,26,4}$ Structural optimization includes several approaches, e.g. material, ${ }^{4}$ size, shape and topology optimization, where size and shape optimization are used for fixed topologies and for improvements of existing structures. Topology optimization, however, is a more general optimization method that can be used in the early conceptual design phase of vehicle development where simplified models often are enough to analyze the structural behavior. An application of this approach is shown in Ref. 25. Using topology optimization methods, the most efficient structures can be found in a relatively early stage of the vehicle design process.

The automotive body structure design problem has been considered in Refs. 55, 22, 20, 21 by applying a sharing penalty vector method in order to define the product platform, i.e., choose which components to share and design the product family with minimal individual variation from the ideal individually optimized designs.

The analytical target cascading process has been applied also to the optimal design of complete vehicle systems. Novel technologies, such as a hybrid-electric propulsion system, in-hub motors, and variable height suspensions were considered in Ref. 54. Emphasis was given to fuel economy, ride, and mobility characteristics.

In order to reduce the product complexity and cost while offering many variants, a widely implemented strategy is the product platform strategy i.e. sharing components and production processes across a platform of products.

Research topics in the automotive field include platform design process development, ${ }^{21,22}$ optimum platform component selection. ${ }^{79}$

Several decision-making models related to the acquisition process of vehicles that address a range of conflicting concerns, such as customer needs, life cycle costs, new technology and design, budget allocation, competitive bidding, and the ability of contractors to meet market targets are discussed in Ref. 12.

\section{F. Integration of vehicle electronic controls}

Basic studies on active suspensions optimization and integration are given in Refs. 36, 102, 65. As discussed previously, simple analytical formulae for the estimation of ride comfort and active safety of vehicles are derived and used to get the optimal controller gains that offer the best compromise among comfort, road holding, and packaging. In Refs. 32, 33 this problem is reformulated by considering the vehicle body mass and the tire radial stiffness as stochastic parameters in order to derive the optimal trade-off solutions in a stochastic framework.

Research in several application areas has unveiled a coupling between the plant (design) and control optimization problems. Due to this coupling, optimizing a plant and control sequentially does not guarantee system optimality. The results have been demonstrated using a simple combined passive/active car suspension case study in Ref. 19.

A method to improve ride and handling of an automobile fitted with four active suspensions (sky hook dampers, active anti-roll bars), active four-wheel steering, and traction control (controlled differential) has been proposed in Ref. 65. The designer is asked to iterate between an innovation stage and a trade-off stage, in order to assess the effectiveness of candidate design issues for the synthesis of new control schemes.

In Ref. 82, a vehicle dynamics integrated control algorithm designed by using a non-linear optimization method is proposed for 4-wheel-distributed steering and 4-wheel-distributed traction/braking systems.

\section{A SIMPLE EXAMPLE}

A simple example is presented in the following to show how very general results can be obtained by applying correctly the optimization procedures introduced above.

The problem is to select the stiffness and damping of a passively suspended vehicle. The vehicle behavior, while running on a randomly profiled road, is described by means of the well known quarter car vehicle model (see Fig. 2a) and the objective is to minimize discomfort (the standard deviation of the acceleration of the body of the vehicle $\left(\sigma_{\ddot{x}_{2}}\right)$, road holding (the standard deviation of the force acting between the road and the wheel $\left(\sigma_{F_{z}}\right)$ and working space (the standard deviation of the relative displacement between the wheel and the vehicle body $\left(\sigma_{x_{2}-x_{1}}\right)$.

Using the symbols denoted in Fig. 2a, we define 


$$
q=\frac{m_{1}}{m_{2}}, K_{x}=k_{2} \frac{(1+q)^{2}}{k_{1} q}, R_{x}=r_{2} \sqrt{\frac{(1+q)^{3}}{k_{1} m_{2} q}}
$$

to derive the analytical expressions of the Pareto-optimal set into the (non-dimensional) design variables domain $\left(K_{x}, R_{x}\right)$ for the three combinations of two objective functions (boundaries of the Pareto-optimal set for the problem with three objective functions, namely $\sigma_{\ddot{x}_{2}}, \sigma_{F_{z}}, \sigma_{x_{2}-x_{1}}$ (see Refs. 35, 33). These are

$$
\begin{aligned}
& \operatorname{optimal}\left(\sigma_{\ddot{x}_{2}}, \sigma_{F_{z}}\right) \rightarrow R_{x}=\sqrt{(1+q) K_{x}-q K_{x}^{2}} \text { with } 0 \leq K_{x} \leq 1 \\
& \operatorname{optimal}\left(\sigma_{\ddot{x}_{2}}, \sigma_{x_{2}-x_{1}}\right) \rightarrow R_{x} \geq 0, K_{x}=0 \\
& \operatorname{optimal}\left(\sigma_{F_{z}}, \sigma_{x_{2}-x_{1}}\right) \rightarrow R_{x} \geq 1, K_{x}=1
\end{aligned}
$$

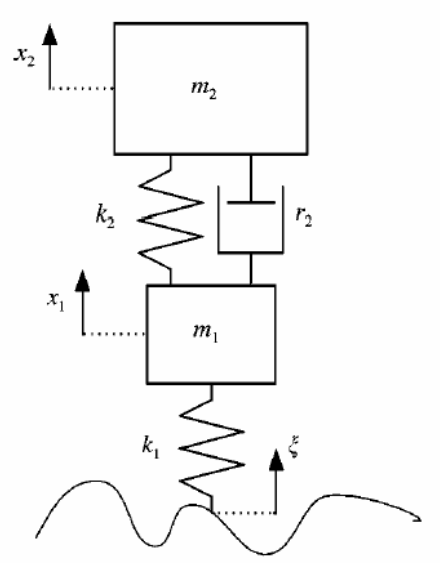

(a)

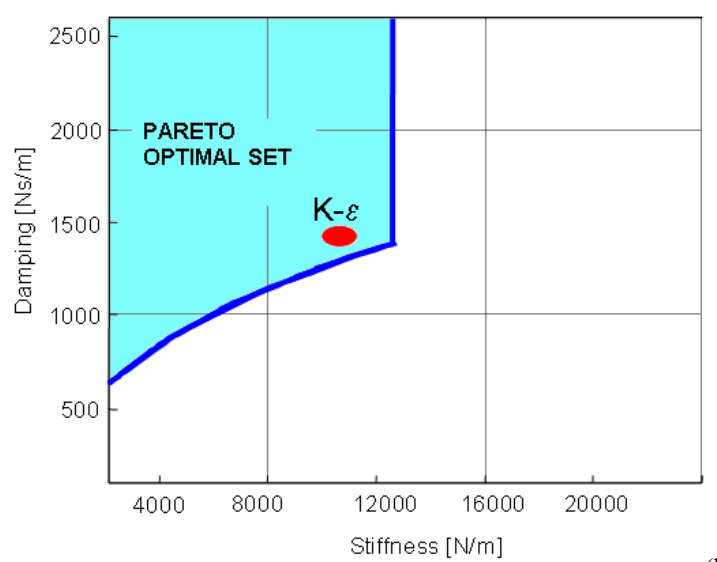

(b)

Fig. 2. Pareto-optimal set (filled area) and ke-optimal solutions (see Ref. 59) for the vehicle passive suspension optimization problem in the design variables space (suspension damping $\mathrm{r}_{2}[\mathrm{Ns} / \mathrm{m}]$, suspension stiffness $\mathrm{k}_{2}[\mathrm{~N} / \mathrm{m}]$ ), vehicle data reported in Ref. 35 .

The designer can get a direct insight into the suspension design problem by inspection of the expressions above. The Pareto-optimal set into the design variable domain (suspension stiffness $k_{2}$ and suspension damping $r_{2}$ ) for the $\sigma_{\ddot{x}_{2}}, \sigma_{F_{z}}, \sigma_{x_{2}-x_{1}}$ problem is the filled area in Fig. $2 \mathrm{~b}$ (reference vehicle data are reported in Ref. 35). The solutions belonging to the Pareto-optimal set are equally desirable from the designer's point of view.

For the selection of the final suspension design solution a ke-optimality approach, extensively described in Ref. 59,

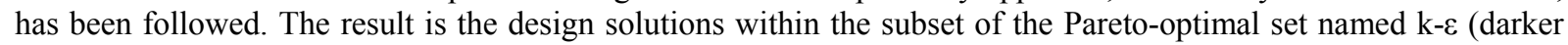
area) in Fig. 2b. The result seems consistent with the solution selected by very skilled suspension specialists, after an expensive amount of trials.

In a stochastic formulation, the vehicle body mass $m_{2}$ and the tire radial stiffness $k_{1}$ can be considered as stochastic parameters due to the variety of possible loading conditions and to the variability of the tire pressure $\left(m_{2}: C V=\right.$ $\sigma / \mu=0.10, k_{1}: C V=0.10$ ). The design variables to be optimized are the stiffness $k_{2}$ and the damping $r_{2}$ of the suspension considered as stochastic design variables due to variations with respect to their nominal value caused by production tolerances and/or wear $\left(k_{2}: C V=0.03, r_{2}: C V=0.10\right)$. The computed Pareto-optimal set is shown in Fig.3. 


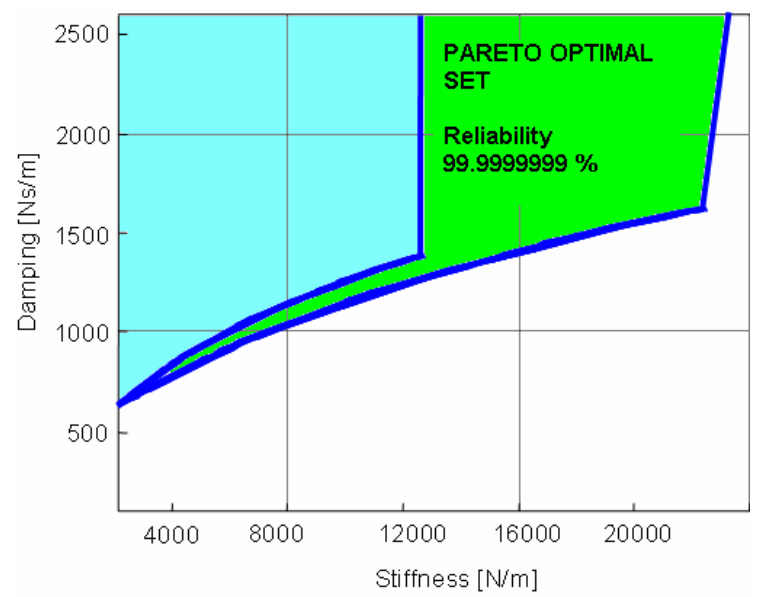

Fig. 3. Pareto-optimal set (filled area) for the vehicle passive suspension optimization problem in the design variables space (suspension damping $\mathrm{r}_{2}[\mathrm{Ns} / \mathrm{m}]$, suspension stiffness $\mathrm{k}_{2}[\mathrm{~N} / \mathrm{m}]$ ), vehicle data reported in Ref. 35. The deterministic solution is reported together with the solution obtainable by applying a reliability-based optimization method.

The figure shows that the increase in robustness (higher reliability) implies a shift of the optimal solutions to higher values of the suspension stiffness $\mathrm{k}_{2}$. It is shown that the Pareto-optimal region computed by imposing a very high reliability (99.9999999 \%) is an extension of the deterministic one. See Ref. 33 for further details.

\section{Conclusion}

The article presented a critical review of the optimization methods used for solving vehicle system design problems. The correct optimization approach discards the "push the button and get the design" idea in favor of a realistic approach that recognizes the role of the designer as the leading force in the design process and the role of computers and software as indispensable tools. Successful optimization requires availability of appropriate analysis models and knowledge of the capabilities and limitations of the mathematical optimization techniques. Most of the modern software available for commercial use are capable of supporting such efforts effectively. However, an increased degree of familiarity is required before more complex studies are undertaken. The inclusion of uncertainty in the problem formulations is a relative recent development in the available optimization tools. Although solution robustness is desirable along with optimality, reliability-based optimal design formulations are substantially more computationally intensive than deterministic ones. Similarly, performing full Pareto optimality studies can be very expensive.

\section{References}

${ }^{1} \mathrm{Ahn}, \mathrm{Y}$. K. et Al. "Optimal design of an engine mount using an enhanced genetic algorithm with simplex method" Vehicle System Dynamics Vol. 43, No. 1, pp. 57 - 81, 2005.

${ }^{2}$ Alleyne, A., and Hedrick, J.K. "Application of nonlinear control theory to electronically controlled suspensions". Vehicle System Dynamics, 22, 1993.

${ }^{3}$ Aminpour, M. et Al. "A Framework for Reliability-based MDO of Aerospace Systems" (AIAA-2002-1476) Proc. of the 43rd AIAA/ASME/ASCE/AHS/ASC Structures, Structural Dynamics, and Materials Conference, Denver, Colorado, April 2002.

${ }^{4}$ Ashby, M.F. "Multi-objective optimization in material design and selection", Acta Materialia, vol 48, pp. 359-369, 2000.

${ }^{5}$ Baumal, A.E., McPhee, J.J., Calamai, P.H. "Application of genetic algorithms to the design optimization of an active vehicle suspension system". Computer Methods in Applied Mechanics and Engineering, 163, pp. 87-94 , 1998.

${ }^{6}$ Belegundu, A.D. and Chandrupatla, T.R., Optimization Concepts and Applications in Engineering, Prenctice Hall, 1999.

${ }^{7}$ Blouin,, V., G. Fadel, I. Haque, J. Wagner, and H. Samuels, "Continuously Variable Transmission. Design for Optimum Vehicle Performance by Analytical Target Cascading", International Journal of Heavy Vehicle Design - Special Automotive Research Center Edition, vol. 11, nos. 3/4, pp. 327-348, 2004.

${ }^{8}$ Caballero R, Cerdá E, Muñoz M, Rey L and Stancu-Minasian I M. "Efficient Solution Concepts and their Relations in Stochastic Multiobjective Programming" Journal of Optimization Theory and Applications, vol. 110, number 1, 2001

${ }^{9}$ Chakarvartula, S., Haque, I., Fadel, G., "A Monte-Carlo Simulation Approach to Heavy Vehicle Design for Good Dynamic Performance in Multiple Scenarios," International Journal of Heavy Vehicle Systems (IJHVS), Vol. 10, No. 1/2, 2003. 
${ }^{10}$ Chan, K.Y., Kokkolaras, M., Papalambros, P., Skerlos, S. J., and Mourelatos, Z., "Propagation of Uncertainty in Optimal Design of Multilevel Systems: Piston-Ring/Cylinder-Liner Case Study", Proceedings of SAE World Congress, Detroit, MI, 2004, paper no. 2004-01-1559.

${ }^{11}$ Cheng, F. and Li, D. "Genetic algorithm development for multi-objective optimization of structures". AIAA Journal, 36(6), 1998.

${ }^{12}$ Cooper, A. B., Georgiopoulos, P., Michalek, J. J., and Papalambros, P. Y., "A simulation-based vehicle design strategy for acquisition and requirements validation", Proceedings of the SAE World Congress, Detroit, MI, March 8-11, 2004.

${ }^{13}$ Cooper, A. B., Kokkolaras, M., and Papalambros, P. Y., "A dual-use enterprise context for vehicle design and technology valuation", Proceedings of the Society of Automotive Engineers World Congress, Detroit, MI, March 8-11, 2004, SAE paper 2004-01-1588.

${ }^{14}$ Dahlberg, T., "An optimized speed-controlled suspension of a 2-DOF vehicle travelling on a randomly profiled road", Journal of Sound and Vibration, vol. 62, 1979

${ }^{15}$ Davis, L. "The Handbook of Genetic Algorithms". Van Nostrand Reingold, New York, 1991.

${ }^{16}$ Deb, K., "A Fast and Elitist Multiobjective Genetic Algorithm: NSGA-II”, IEEE transactions on Evolutionary Computation, Vol.6, No. 2, pp.182-197, 2002.

${ }^{17}$ De Weck, O. "Multiobjective Optimization: History and Promise", Keynote Paper, Third China-Japan-Korea Joint Symposium on Optimization of Structural and Mechanical Systems, Kanazawa, Japan, October 30-November 2, 2004.

${ }^{18}$ Ehrgott, M. and Wiecek, M.M. Multiobjective Programming, in: Multiple Criteria Decision Analysis: State of the Art Surveys, J. Figueira, S. Greco and M. Ehrgott (eds.), Springer, New York, pp. 667-722, 2005.

${ }^{19}$ Fathy, H., Papalambros, P., Ulsoy, A., Hrovat, D. "Nested Plant Controller Optimization with Application to Combined Passive/Active Automotive Suspensions" Proceedings of the American Control Conference, Denver, Colorado June 4-6, 2003.

${ }^{20}$ Fellini R., Papalambros P.Y., and Weber T., "Application of a Product Platform Design Process to Automotive Powertrains," Proceedings of the 8th AIAA/NASA/USAF/ISSMO Symposium on Multidisciplinary Analysis and Optimization, Paper No. 4849, Long Beach, CA, 2000.

${ }^{21}$ Fellini, R. et Al. "A Sensitivity-based Commonality Strategy for Family Products of Mild Variations, with application to automotive body structures" 9th AIAA/ISSMO Symposium on Multidisciplinary Analysis and Optimization, 4-6 September 2002 (AIAA 2002-5610).

${ }^{22}$ Fellini, R., Kokkolaras, M., and Papalambros, P., "Quantitative Platform Selection in Optimal Design of Product Families, with Application to Automotive Engine Design ",, To appear in Journal of Engineering Design, 2004.

${ }^{23}$ Fenyes, P. A., Donndelinger, J., Bourassa, J., "A New System for Multidisciplinary Analysis and Optimization of Vehicle Architectures", AIAA paper, AIAA-2002-5509, 2002.

${ }^{24}$ Filipi, Z. et Al. "Combined optimization of design and power management of the hydraulic hybrid propulsion system for the $6 \times 6$ medium" Int. J. of Heavy Vehicle Systems, Vol. 11, Nos 3/4, 2004.

${ }^{25}$ Fredricson H. "Property Optimization of Vehicle Body Structure Elements using Simplified Models". Proc. of the WCSMO5, Lido di Jesolo, May 2003.

${ }^{26}$ Fredricson, H., Johansen, T., Klarbring A., Petersson, J. "Topology optimization of frame structures with flexible joints" Journal of Structural and Multidisciplinary Optimization", vol.3, 2003.

${ }^{27}$ Fujita, K., et Al.: Design Optimization of a Multilink Suspension System for Total Vehicle Handling and Stability. Proc. of the 7th AIAA/USAF/NASA/ISSMO Symposium on Multidisciplinary Analysis and Optimization, St. Louis, 1998.

${ }^{28}$ Gobbi, M., Haque, I., Papalambros, P., Mastinu G. "Optimization and integration of ground vehicle systems" Vehicle System Dynamics Vol. 43, No. 6-7, June-July 2005.

${ }^{29}$ Gobbi M. and Mastinu, G., "Global Approximation: Performance Comparison of Different Methods, with an Application to Road Vehicle System Engineering", in "Innovation in Vehicle Design and Development", (I. Haque et al. Eds.), DE-Vol. 101, ASME, pp. 15-24. New York, 1999.

${ }^{30}$ Gobbi, M. and Mastinu, G. "Stochastic Multi-Objective Optimization for the Design of Vehicle Systems". Proc. of the WCSMO5 International Conference, Lido di Jesolo, May, 2003.

${ }^{31}$ Gobbi, M. et Al. "Optimal \& Robust Design of a Road Vehicle Suspension System”. Vehicle System Dynamics Supplement 33, pp. 3-22. 1999.

${ }^{32}$ Gobbi, M., Levi, F., Mastinu, G., "Multi Objective Robust Design of the Suspension System of Road Vehicles", Vehicle System Dynamics Supplement 41:537-546, 2004.

${ }^{33}$ Gobbi, M., Levi, F., Mastinu, G., "Multi-objective stochastic optimisation of the suspension system of road vehicles", Journal of Sound and Vibration, 2006.

${ }^{34}$ Gobbi, M., Mastinu, G., "On the optimal design of composite material tubular helical springs ”, Meccanica, vol. 304, 2001

${ }^{35}$ Gobbi, M., Mastinu, G., "Symbolic description and optimization of the dynamic behaviour of vehicles running on rough road", Journal of Sound and Vibration, Vol. 245, No. 3, August, 2001.

${ }^{36}$ Gobbi, M., Mastinu, G., "Symbolical multi-objective optimization of the dynamic behaviour of actively suspended road vehicles", Int. Journal of Vehicle Design, Vol. 28, Nos. 1/2/3, 2002

${ }^{37}$ Gobbi, M., Mastinu, G., Caudano, M., "Stochastic Multi-objective Optimization of a Gearbox Synchroniser and Selector Mechanism", Proceedings of the ASME IMECE 2003, Volume 2, November, 2003, Washington, D.C. USA, ISBN 0-7918-46644

${ }^{38}$ Gobbi, M., Mastinu, G., D’Orazio, A., Caudano, M., Faustini, G., “On the Optimization of a Double Cone Synchroniser for Improved Manual Transmission Shiftability” Advanced Vehicle Technologies DE Vol. 103 ASME 2002 (ISBN 0-7918-1692-3) 
${ }^{39}$ Gobbi, M., Mastinu, G., Doniselli, C., “Optimising a car chassis”, Vehicle System Dynamics, vol.32, no.2-3, 1999, pp. 149170 .

${ }^{40}$ Gobbi, M. "Optimal and Robust Design of Ground Vehicle Systems" Proceedings of ESDA2006, 8th Biennial ASME Conference on Engineering Systems Design and Analysis, July 4-7, Torino, 2006.

${ }^{41}$ Goldberg, D. Genetic Algorithms in Search, Optimization, and Machine Learning. Addison-Wesley, 1989.

${ }^{42}$ Goncalves, J., Ambrosio, J. "Optimization of Vehicle Suspension Systems for Improved Comfort of Road Vehicles Using Flexible Multibody Dynamics" Nonlinear Dynamics 34: 113-131, 2003.

${ }^{43}$ Goncalves, J., Ambrosio, J. "Road Vehicle Modeling Requirements for Optimization of Ride and Handling" Multibody System Dynamics, 13, pp.3-23, 2005.

${ }^{44}$ Grierson, D.E. and Hajela, P. editors. Emergent Computing Methods in Engineering Design. Applications of Genetic Algorithms and Neural Networks. Springer Verlag, 1996.

${ }^{45} \mathrm{Gu}$, L., Yang, R.J., Cho, C.H., Makowski, M., Faruque, M., Li, Y. "Optimization and robustness for crashworthiness" International Journal of Vehicle Design 26(4), 2001.

${ }^{46}$ Hamza, K., Hossoy, I., Reyes-Luna, J. F., and Papalambros, P. Y., "Combined Maximization of Interior Comfort and Frontal Crashworthiness in Preliminary Vehicle Design", International Journal of Vehicle Design, Vol. 35, No. 3, 2004, pp. 167-185.

${ }^{47}$ Hedrick, J.K. Introduction to Active Suspensions. CCG Course on Control of Vehicle Ride and handling, July 1990.

${ }^{48}$ Holland, J. Adaptation in Natural and Artificial Systems. The University of Michigan Press, Ann Arbor, 1975.

${ }^{49}$ Hrovat, D. "Applications of optimal control to advanced automotive suspension design". Transactions of the ASME, Journal of Dynamic Systems, Measurement, and Control 115, June 1993.

${ }^{50}$ Karnopp, D., and Trikha, A.. "Comparative Study of Optimization Techniques for Shock and Vibration Isolation" Transaction of the ASME, Journal of Engineering for Industry 91, 4, 1969.

${ }^{51}$ Kim, H., Rideout D., Papalambros, P., Stein, J. "Analytical Target Cascading in Automotive Vehicle Design”, Journal of Mechanical Design, Vol. 125, pag. 481, September 2003.

${ }^{52}$ Kirkpatrick, S., Gelatt, C.D., Vecchi, M.P. “Optimization by Simulated Annealing”. Science 13, 1983, volume 220, number 4598.

${ }^{53}$ Kodiyalam, S., Yang, R. J. , Gu, L., Tho, C., "Large-Scale, Multidisciplinary Optimization of a Vehicle System in a Scalable, High Performance Computing Environment" Proceedings of DETC2001 ASME Design Engineering Technical Conferences September 9-12, 2001, Pittsburg, Pennsylvania, USA.

${ }^{54}$ Kokkolaras, M. et Al. "Simulation-based optimal design of heavy trucks by model-based decomposition: An extensive analytical target cascading case study". Int. J. of Heavy Vehicle Systems, Vol. 11, Nos 3/4, 2004.

${ }^{55}$ Kokkolaras, M., Fellini, R., Kim, H.M., Michelena, N.F. and Papalambros, P.Y. "Extension of the target cascading formulation to the design of product families". Journal of Structural and Multidisciplinary Optimization, 24(4):293-301, 2002.

${ }^{56}$ Kokkolaras, M., Mourelatos, Z. P., and Papalambros, P. Y., "Design Optimization of Hierarchically Decomposed Multilevel System under Uncertainty", Proceedings of the ASME 2004 Design Engineering Technical Conferences, Salt Lake City, Utah, September 28 - October 2, 2004, DETC2004/DAC-57357.

${ }^{57}$ Kokkolaras, M., Mourelatos, Z.P., and Papalambros P.Y., "Design Optimization of Hierarchically Decomposed Multilevel Systems under Uncertainty", Proceedings of 2004 ASME Design Engineering Technical Conferences: 30th Design Automation Conference, Salt Lake City, UT, 2004, paper no. DETC2004/DAC57357.

${ }^{58}$ Levi, F., Gobbi, M. and Mastinu, G., "An application of multi-objective stochastic optimization to structural design", Structural and Multidisciplinary Optimization, Springer-Verlag, No. 10, 2004, DOI: 10.1007/s00158-004-0456-2.

${ }^{59}$ Levi, F., Gobbi, M., Mastinu, G., Farina, M., "Multi-Objective design and selection of one optimal solution", Proc. of the ASME IMECE 2004 Int. Conference, Anaheim, Nov 2004.

${ }^{60}$ Li, Z., Georgiopoulos, P., Papalambros, P. Y., Filipi, Z., Wu, G., and Yang, X., "Model Based Analysis of Performance-Cost Tradeoffs for Engine Manifold Surface Finishing", Proceedings of the Society of Automotive Engineers World Congress, Detroit, MI, March 8-11, 2004, SAE papaer 2004-01-1561.

${ }^{61} \mathrm{Li}$, Z., Kokkolaras, M., Jung, D., Papalambros, P. Y., and Assanis, D. N., "An Optimization Study of Manufacturing Variation Effects on Diesel Injector Design with Emphasis on Emissions", Proceedings of the SAE World Congress, Dearborn, MI, March 8-11, 2004, SAE paper 2004-01-1560.

${ }^{62}$ Longo, R., Anedda, G., Testa, M. "Integrated Multibody Structural Optimization for Car Suspension Design" Proc. of the TCN CAE 2003 International Conference, Cagliari, 2003

${ }^{63}$ Mastinu, G. "Automotive Suspension Design by Multiple Objective Programming" Proceedings of the International Symposium on "Advanced Vehicle Control" (AVEC'94), JSAE, Tsukuba, Japan, 1994, pp. 73-78.

${ }^{64}$ Mastinu, G. and Gobbi, M. "Advances in the optimal design of mechanical systems". Course coordinated by CISM (International Centre for Mechanical Sciences), web: www.europeindia.org, Birla Science Centre, Hyderabad, 1999.

${ }^{65}$ Mastinu, G., Integrated Controls and Interactive Multiple Objective Programming for the Improvement of Ride and Handling of Road Vehicles. In Pawelussen, J.P., Pacejka, H. (Ed.) 'Smart Vehicles' Swets \& Zeitlinger, Lisse, The Netherlands, 1995

${ }^{66}$ Mastinu, M. Gobbi, M., Mimini, M. "A Parameter Identification Method for Road Accidents Reconstruction" Proceedings of ASME IMECE 2003, Volume 2, Novembre, 2003, Washington, D.C. USA, ISBN 0-7918-4664-4.

${ }^{67}$ Mastinu, M. Gobbi, M., Miano C., Optimal Design of Complex Mechanical Systems with Applications to Vehicle Engineering. Springer Verlag, (2006).

${ }^{68}$ Metropolis, N. and Rosenbluth, A. and Rosenbluth, R. and Teller, A. and Teller, E. "Equation of state calculations by fast computing machines" Journal of Chemical Physics, 21 pag.1087-1092, 1953. 
${ }^{69}$ Matusov, J.B., Multicriteria Optimization and Engineering, Chapman \& Hall, New York ,1995.

${ }^{70}$ Miano, C., Gobbi, M. and Mastinu, G. "A tutorial on present and future applications of global approximation issues with application to vehicle design problems”. In DE-Vol. 112 Advanced Vehicle Technologies. ASME, 2001.

${ }^{71}$ Miano, C., Gobbi, M., Mastinu, G., "Multi-Objective Optimization of the Handling Performances of a Road Vehicle: A Fundamental Study on Tire Selection”, ASME Journal of Mechanical Design, July 2004, Vol. 126 pag. 687.

${ }^{72}$ Miano, C., Gobbi, M., Mastinu, G., Cesarini, R., "On the integrated design of the tyre-suspension system of a racing car", in “Advanced vehicle technologies”, (I. Haque et al. Eds.), ASME Publication, DE-vol. 106, New York, 2000

${ }^{73}$ Miano, C., Optimization methods of complex mechanical system with particular reference to ground vehicles (In Italian). PhD thesis, Politecnico di Milano, 2003.

${ }^{74}$ Michelena, N.F. and Papalambros., P.Y. "A Hypergraph Framework for Optimal Model-Based Decomposition of Design Problems". Journal of Computational Optimization and Applications, 2, Volume 8, 1997.

${ }^{75}$ Michelena, N.F., Park, H., and Papalambros, P.Y. "Convergence properties of analytical target cascading”. AIAA Journal, 41(5):897-905, May 2003.

${ }^{76}$ Miettinen, K., Nonlinear Multiobjective Optimization, Kluwer Academic Publishers, Boston, 1999.

${ }^{77}$ Mitschke, M. Dynamik der Kraftfahrzeuge. Springer Verlag, Berlin, 1990.

${ }^{78}$ Nakhaie Jazar, G., Alkhatib, R. and Golnaraghi, M.F. "Root mean square optimization criterion for vibration behaviour of linear quarter car using analytical methods”, Vehicle System Dynamics Vol. 44, No. 6, pp. 477-512, 2006.

${ }^{79}$ Nelson, S. A., II, Parkinson, M. B. and Papalambros, P. Y. "Multicriteria optimization in product platform design" Journal of Mechanical Design, 123(2), 199-204, 2001.

${ }^{80} \mathrm{Ng}$, W.Y. Interactive Multi-Objective Programming as a framework for Computer Aided Control Systems Design. Springer Vlg, Berlin, 1989.

${ }^{81}$ Nishiwaki, S., Nishigaki, H., Tsurumi, Y., Kojima, Y., Kikuchi, N., First Order Analysis - New CAE Tool for Automotive Body Designers, SAE paper, 2001-01-0768, 2001.

${ }^{82}$ Ono, E., Hattori,Y., Muragishi, Y. and Koibuchi, K., "Vehicle dynamics integrated control for four-wheel-distributed steering and four-wheel-distributed traction/braking systems”, Vehicle System Dynamics, Vol. 44, No. 2, pp. 139-151, 2006.

${ }^{83}$ Papalambros, P.P. and Wilde, D.J., Principles of Optimal Design: Modeling and Computation, Cambridge University Press, New York, 2000.

${ }^{84}$ Pardalos, P.M., Resende, M., Pardalos, P., Handbook of Applied Optimization. Oxford University Press, 2002.

${ }^{85}$ Ruzika, S. and Wiecek, M.M. "A survey of approximation methods in multiobjective programming", Journal of Optimization Theory and Applications 126:(3), 2005.

${ }^{86}$ Scarlat, G., Haque, I., Fadel, G. and Schuller, J., "A Modified Monte-Carlo Approach To Simulation-Based Vehicle Design With Multiple Objectives And Multiple Scenarios," Proc of the SAE World Congress, 2002

${ }^{87}$ Schäffer, J., Some Experiments in Machine Learning Using Vector Evaluated Genetic Algorithms. PhD thesis, Vanderbilt University, Nashville, 1984.

${ }^{88}$ Schuller, J., Haque, I. and Fadel, G., "Methods for Simulation-Based Optimization of Vehicle Handling Behavior," Advanced Vehicle Technologies, I. Haque and M. El-Gindy, Editors, November, 2000.

${ }^{89}$ Schuller, J., Imtiaz Haque, and Marc Eckel, " An Approach for Optimization of Vehicle Handling Behaviour in Simulation," Dynamics of Vehicles on Roads and Tracks, Supplement to Vehicle System Dynamics, August, 2001.

${ }^{90}$ Sharp, R.S. and Crolla, D.A. "Road Vehicle suspension system design - a review". Vehicle System Dynamics, $16,1987$.

${ }^{91}$ Sinha, K. "Reliability-based multi-objective optimization methodology for engineering systems" Proceedings of ESDA2006, 8th Biennial ASME Conference on Engineering Systems Design and Analysis, July 4-7, Torino, 2006.

${ }^{92}$ Siddal J N. "A New Approach to Probability in Engineering Design and Optimization". ASME J. of Mechanisms, Transmissions, and Automation in Design, 1984, 106:5-10

${ }^{93}$ Srinivas, N. and Deb., K. "Multiobjective optimization using nondominated sorting in genetic algorithms". Evolutionary Computation, (2(3)), 1994.

${ }^{94}$ Suh, M.-W., Le, J.-H., Cho, K.-Y. and Kim, S.-I., "Section property method and section shape method for the optimum design of a vehicle body structure", Int. Journal of Vehicle Design, Vol. 30, 2002.

${ }^{95}$ Suppapitnarm A., Seffen K. A., Parks G. T., Clarkson P. J., and Liu J. S., "Design by multiobjective optimization using simulated annealing" Proc. of the International conference on engineering design ICED, Munich, Germany, 1999.

${ }^{96}$ Tangella, C., "Simulated Annealing as an Optimization Tool for Multi-Criteria, Multi-Scenario Optimization," MS Thesis, Clemson University, May 2003.

${ }^{97}$ Tezuka, D. Uniform Random numbers: Theory and Practice. Kluwer Academic publishers, 1995.

${ }^{98}$ Thompson, A. "An active suspension with optimal linear state feedback". Vehicle System Dynamics, no. $5,1976$.

${ }^{99}$ Thompson, A. "Suspension design for optimum road-holding". SAE Paper 830663, 1983.

${ }^{100}$ Youn B.D, Choi, K.K., Kokkolaras, M., Papalambros, P.Y., Mourelatos, Z.P., Gorsich, D., "Techniques for Estimating Uncertainty Propagation in Probabilistic Design of Multilevel Systems". 10th AIAA/ISSMO Multidisciplinary Analysis and Optimization Conference 30 August - 1 September, Albany, New York (AIAA 2004-4470), 2004.

${ }^{101}$ Youn, B.D., Choi, K.K, “Selecting Probabilistic Approaches for Reliability-based design optimization”, AIAA, Vol. 42, No. $1,2004$.

${ }^{102}$ Zaremba, A., Hampo, R., and Hrovat, D. "Optimal active suspension design using constrained optimization". Journal of Sound and Vibration, vol. 207, no. 3, 1997. 